OPEN ACCESS

Edited by:

Shuji Mizumoto,

Meijo University, Japan

Reviewed by:

Shinji Miyata,

Tokyo University of Agriculture and Technology, Japan

Jerry Silver,

Case Western Reserve University,

United States

${ }^{*}$ Correspondence:

Kazuma Sakamoto

sakamoto@med.nagoya-u.ac.jp

Specialty section:

This article was submitted to

Signaling,

a section of the journal

Frontiers in Cell and Developmental

Biology

Received: 29 April 2021

Accepted: 25 May 2021

Published: 16 June 2021

Citation:

Sakamoto K, Ozaki T and

Kadomatsu K (2021) Axonal

Regeneration by Glycosaminoglycan.

Front. Cell Dev. Biol. 9:702179.

doi: 10.3389/fcell.2021.702179

\section{Axonal Regeneration by Glycosaminoglycan}

\author{
Kazuma Sakamoto ${ }^{1,2 *}$, Tomoya Ozaki ${ }^{1}$ and Kenji Kadomatsu ${ }^{1,2}$ \\ ${ }^{1}$ Department of Biochemistry, Nagoya University Graduate School of Medicine, Nagoya, Japan, ${ }^{2}$ Institute for Glyco-Core \\ Research (iGCORE), Nagoya University, Nagoya, Japan
}

Like other biomolecules including nucleic acid and protein, glycan plays pivotal roles in various cellular processes. For instance, it modulates protein folding and stability, organizes extracellular matrix and tissue elasticity, and regulates membrane trafficking. In addition, cell-surface glycans are often utilized as entry receptors for viruses, including SARS-CoV-2. Nevertheless, its roles as ligands to specific surface receptors have not been well understood with a few exceptions such as selectins and siglecs. Recent reports have demonstrated that chondroitin sulfate and heparan sulfate, both of which are glycosaminoglycans, work as physiological ligands on their shared receptor, protein tyrosine phosphatase sigma (PTP $)$ ). These two glycans differentially determine the fates of neuronal axons after injury in our central nervous system. That is, heparan sulfate promotes axonal regeneration while chondroitin sulfate inhibits it, inducing dystrophic endbulbs at the axon tips. In our recent study, we demonstrated that the chondroitin sulfate (CS)-PTPo axis disrupted autophagy flux at the axon tips by dephosphorylating cortactin. In this minireview, we introduce how glycans work as physiological ligands and regulate their intracellular signaling, especially focusing on chondroitin sulfate.

Keywords: chondroitin sulfate, heparan sulfate, axonal regeneration, PTP $\sigma$, autophagy, dystrophic endbulb

\section{INTRODUCTION}

The human neural circuit, composed of the central nervous system (CNS) and the peripheral nervous system (PNS), reaches approximately 1 million $\mathrm{km}$ and is formed mainly by neural axons. An axon is a structure that is elongated from the cell body and relays information to its target cells, including neurons, glands, and muscles, by conducting electrical pulses and releasing neurotransmitters.

Axonal injury to our mature CNS, including spinal cord injury and traumatic brain injury, is still an untreatable condition, even with current medical knowledge. It was already recognized and described as "incurable" in the Edwin Smith Surgical Papyrus published between 2,500 and 1,900 BC in Egypt (Hughes, 1988). Emerging evidence has revealed that the lack of trophic factors and the existence of inhibitory environmental cues in the adult CNS made it difficult for nerve axons to spontaneously regenerate. Once damaged, axons enter a dormant state. Regeneration is possible only under certain circumstances.

Upon injury, the distal parts of axons that are separated from the cell body, undergo Wallerian degeneration. The fragmented and degenerated axonal shafts are phagocytosed and removed by microglia or macrophages. The process seems to be important for the regeneration of axons by the surviving neurons. In PNS, Wallerian degeneration is mediated mainly by peripheral macrophages and is accomplished quickly and completely, which is thought to be a factor in the high regeneration capacity of PNS axons. In the CNS, on the other hand, Wallerian degeneration is largely delayed 
and finished incompletely, probably because of the poor phagocytic capacity of microglia, major phagocytic cells in the CNS. In the 1980s, Aguayo and his colleagues clearly demonstrated that CNS axons regenerated through the implanted sciatic nerve "bridge," confirming that the difference in environmental cues between the CNS and the PNS defined each axon's regeneration capability (David and Aguayo, 1981; Benfey and Aguayo, 1982).

In addition to Wallerian degeneration, matrix remodeling after injury largely differs between the CNS and the PNS. In the CNS, the lesion site is surrounded by activated astrocytes (reactive astrocytes), forming a so-called glial scar (Silver and Miller, 2004). This scar is important for fixing the damaged blood-brain barrier (BBB), covering the lesion, protecting tissues from infections, and secreting various regeneration factors (Anderson et al., 2016). However, the glial scar also produces chondroitin sulfate (CS) proteoglycans, which are molecules that inhibit axonal regeneration (Snow et al., 1990, 1991; Rauch et al., 1991; Asher et al., 2000). When the regenerating axon tip makes contact with the CS proteoglycan (CSPG), it stops further extension and enters a dormant state called the dystrophic endbulb.

In this review, we briefly summarize how glycans work as physiological ligands and regulate axonal regeneration by mediating intracellular signaling.

\section{DYSTROPHIC ENDBULB}

In 1928, Santiago Ramon Cajal found swollen axonal tips with multiple vacuoles in a lesion of a canine spinal cord. He reported that the structure was closely associated with poor axonal regeneration ability in the CNS. That structure is now recognized as the dystrophic endbulb or the dystrophic endball. The dystrophic endbulb contains a disorganized cytoskeleton and accumulations of membrane. It is formed acutely after injury can persist for several decades at lesions in human patients (Ruschel et al., 2015). Therefore, the structure was regarded as a therapeutic target for traumatic CNS injury, as Cajal suggested. However, the cellular and molecular mechanisms underlying the formation of dystrophic endbulb were unclear. Silver and his colleagues demonstrated that the increasing concentration gradient of CS that mimicked the in vitro glial scar was sufficient to induce dystrophic endbulbs on cultured adult dorsal root ganglion neurons (Tom et al., 2004). Defects in lysosomal secretion, including that of Cathepsin B, which might be important for matrix degradation and axonal elongation, were also suggested to be a characteristic of dystrophic endbulb (Tran et al., 2018; Tran and Silver, 2021).

\section{ROLES OF GLYCOSAMINOGLYCANS IN CNS}

CS is a glycosaminoglycan (GAG) and an unbranched polymer chain, which consists of the repeating disaccharide unit, glucuronic acid-N-acetylgalactosamine (GlcA-GalNAc)
(Figure 1; Margolis and Margolis, 1993; Kadomatsu and Sakamoto, 2014; Sakamoto and Kadomatsu, 2017). In addition to CS, GAG contains heparan sulfate (HS), keratan sulfate, and hyaluronan. Except for HA, GAG is modified with sulfate groups and covalently attached to specific core proteins, forming proteoglycan (PG). HS is a linear polysaccharide and consists of repeating disaccharide unit, uronic acid and $N$-acetylglucosamine. The sequential modifications, $\mathrm{N}$-deacetylation of $\mathrm{N}$-acetylglucosamines, $\mathrm{N}$-sulfation of glucosamines, and $\mathrm{O}$-sulfations at the $\mathrm{C} 2$-position of uronic acids as well as C3- and/or C6-position of glucosamines can be occurred. Regarding its roles in axonal elongation and its inhibition, HS, which is a linear polysaccharide of repeated disaccharide of uronic acid and was revealed to be a positive regulator (Wang and Denburg, 1992; Aricescu et al., 2002). For example, mice lacking EXT1, one of the essential enzymes for the synthesis of HS, showed abnormal commissure formation of the corpus callosum (Inatani et al., 2003). On the other hand, CS and keratan sulfate were revealed to be negative regulators in axonal elongation. In addition to dystrophic endbulb-forming activity in vitro as described above, several works based on chondroitinase ABC, a CS-degrading enzyme with bacterial origin, clearly demonstrated that CS was involved in the inhibition of axonal regeneration after injury in vivo. Enzymatic digestion of CS side chains on PG by the enzyme dramatically enhanced both anatomical and functional plasticity after various SCI models (Moon et al., 2001; Bradbury et al., 2002). Importantly, combined with intermittent hypoxia, chondroitinase $\mathrm{ABC}$ promoted robust restoration of ventilation after SCI, the impairment of which is a major cause of mortality in human patients (Warren et al., 2018). A recent work also showed that $\mathrm{C}-\mathrm{ABC}$ improved both anatomical and functional outcomes after spinal cord hemisection in monkey (Rosenzweig et al., 2019). Keratan sulfate was also demonstrated to inhibit axonal regeneration both in vitro and in vivo (Snow et al., 1990; Smith-Thomas et al., 1994; Ito et al., 2010; Imagama et al., 2011). It is noteworthy that CS and keratan sulfate often share proteoglycans, such as aggrecan and phosphacan (Rauch et al., 1991; Margolis and Margolis, 1993).

CS is a long glycan chain with approximately 50-100 saccharides on average. The $\mathrm{C} 2$-position of GlcA and the C4- and/or C6-positions of GalNAc can be sulfated, resulting in different disaccharide compositions including A-unit (GlcA-GalNAc4S), C-unit (GlcA-GalNAc6S), D-unit (GlcA2SGalNAc6S), and E-unit (GlcA-GalNAc4S,6S), where 2S, 4S, and $6 \mathrm{~S}$ stand for 2- O-, 4- O-, and 6-O-sulfate, respectively. Thus, a single chain of CS is heterogeneous in both length and structure and has been proposed to contain a "functional domain" to interact with other specific molecules (Mikami et al., 2009; Dickendesher et al., 2012). In mammalian CNS, CSPGs, neurocan, versican, brevican, aggrecan, NG2, and phosphacan, contribute to assemble into an extracellular matrix. Along with the development of the CNS, CS is enriched especially around inhibitory neurons and synapses in a well-organized manner, in what is known as a "perineurnal net," where it regulates synaptic plasticity (Pizzorusso et al., 2002, 2006; Frischknecht et al., 2009; Gogolla et al., 2009; Miyata et al., 2012). Upon injury, 


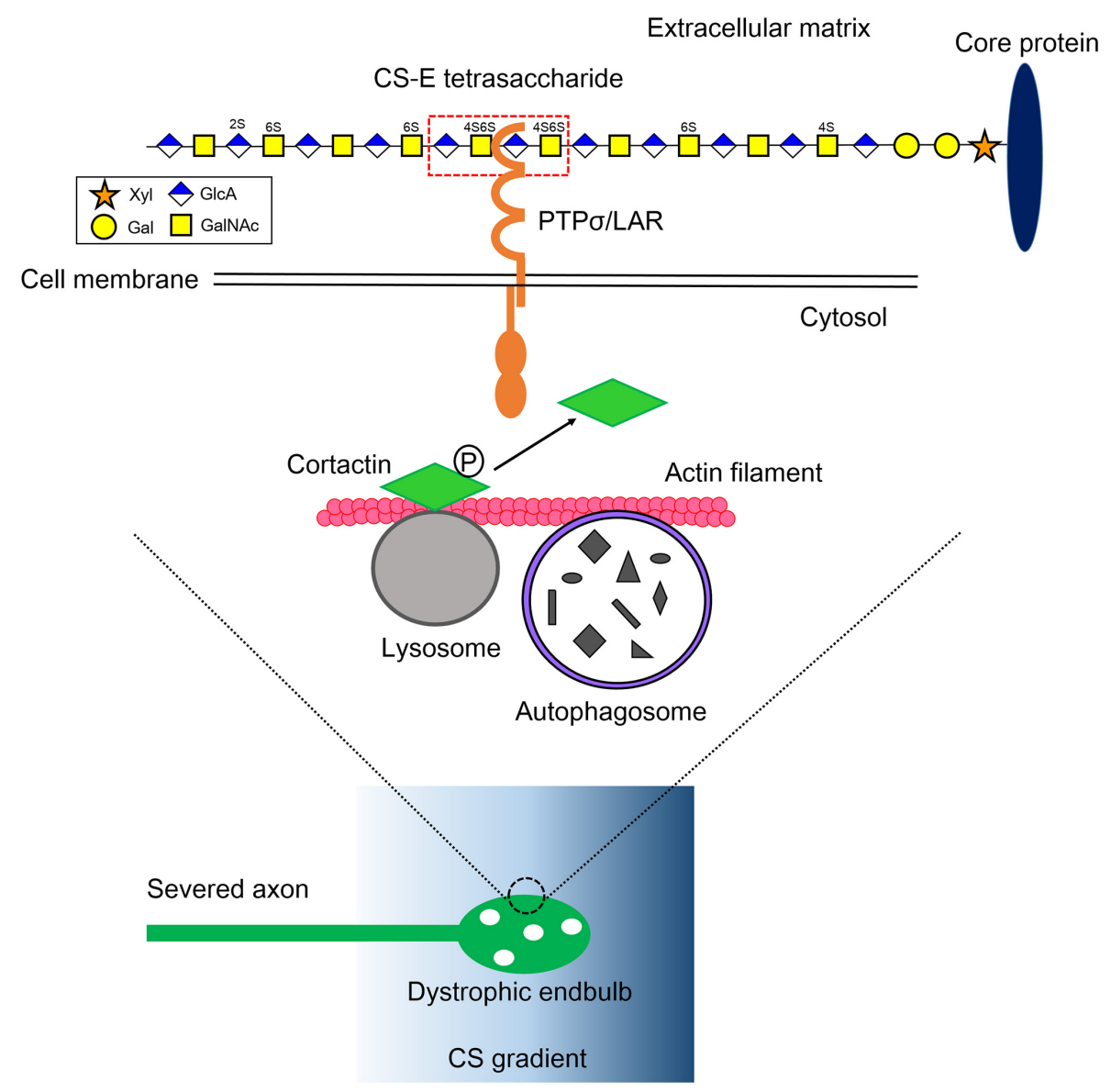

FIGURE 1 | Mechanistic insight into the formation of dystrophic endbulb by CS. The severed axons run into the gradient of CS originated from the perineuronal net and the reactive astrocyte in the lesion. The CS-E tetrasaccharide segment, which rarely appears on a CS chain, preferentially monomerizes and activates its axonal receptor PTPo/LAR. Contrary, HS protects the formation of dystrophic endball by inducing clustering of the receptors. RPTP dephosphorylates cortactin, which is localized onto the lysosome surface and stabilizes actin fibers to facilitate autolysosome formation. As a consequence, the CS-RPTP axis disrupts autophagy flux, inducing the failure of fusion between autophagosomes and lysosomes, and thus abnormal accumulation of autophagosomes. That leadsto transforms of axon tips to dystrophic endbulb. Xyl, xylose; Gal, galactose; GlcA, glucuronic acid; GalNAc, N-acetylgalactosamine; P, tyrosine phosphorylation.

this organized CS matrix might be disrupted and diffused in a disorganized manner and, together with CS newly synthesized by the glial scar, inhibits regeneration of the dissected axons, transforming the dystrophic endbulb at its tip (Silver and Miller, 2004). However, the action mechanisms of CS on axons remained unclear for about two decades after CS was recognized as a major inhibitory cue for axonal regeneration in our CNS. This was largely because specific neuronal receptors for CS had not been identified.

\section{PROTEIN TYROSINE PHOSPHATASE SIGMA FUNCTIONS AS A CS RECEPTOR}

It was a big surprise that protein tyrosine phosphatase sigma (PTP $\sigma)$ and leukocyte common antigen-related (LAR) were identified as neuronal receptors for CS (Shen et al., 2009; Fisher et al., 2011), because these two molecules had already been reported as receptors for HS and found to be positively involved in axon guidance (Aricescu et al., 2002; Johnson et al., 2006). Both PTP $\sigma$ and LAR, together with $\mathrm{PTP} \delta$, belong to the type IIa RPTP (receptor-type protein tyrosine phosphatase) family (Tonks, 2006). They are type I transmembrane proteins that each possess three immunoglobulin-like domains and typically four or eight fibronectin repeats. Two tandem repeats of the PTP domain composed of catalytically active D1 and inactive D2 form the intracellular domain (Tonks, 2006). The catalytic activity is regulated by receptor monomerization and multimerization. As a monomer, the D1 domain is free and active, while the D1 domain is cis-inhibited by the D2 domain as a multimer. Both CS and HS share the same binding domain on the first immunoglobulinlike domain of PTP $\sigma$ and LAR, in which the basic amino acids form clusters at the surface, implying its ionic interaction with the sulfate groups of CS and HS (Aricescu et al., 2002; Shen et al., 2009). The additional binding site at the juxtamembrane domain on PTP $\sigma$ for HS was also reported (Katagiri et al., 2018). On the basis of biochemical and structural analyses, the "switch model" of PTP $\sigma$ by HS and CS was proposed, in which HS 
induced receptor clustering, on the other hand, CS promoted receptor monomerization (Coles et al., 2011). However, how these two sulfated glycans act in such an opposing manner remained unclear, as did the specific substrates for PTP $\sigma$ that are responsible for forming the dystrophic endbulb.

\section{DISRUPTION OF AUTOPHAGY IN DYSTROPHIC ENDBULB BY CS}

In our recent work, we tried to explore deeply how CS and HS differentially regulate PTP $\sigma$, which ultimately determines axonal fate after injury (Sakamoto et al., 2019). The heterogeneity of glycan sulfation patterns in a single chain of GAG makes it harder to determine which segment binds to PTP $\sigma$. To overcome this, we synthesized and prepared a pure CS and HS oligosaccharides library with defined lengths and sulfation patterns. The surface plasmon resonance method was initially used to determine the interactions between CS variants and PTPб. We found that CS-E, which has a highly proportion of E-unit (GlcA-GalNAc4S,6S), showed the best affinity to PTPo among the CS variants, CS-A, CS-C, CS-D, and CS-E (Sakamoto et al., 2019). We found that CS-E, which has C4and C6-sulfation on GalNAc residues, showed the best affinity to PTP $\sigma$. We then tested PTP $\sigma$ with different lengths of CS$\mathrm{E}$ and determined that the minimal binding segment of CS-E was a tetrasaccharide (Figure 1; Sakamoto et al., 2019). It is noteworthy that CS-E is a rare sulfation pattern and accounts for only a small percentage of the total CS in the injuredCNS in mice (Properzi et al., 2005). This means the frequency of CS-E tetrasaccharide can be estimated to be one at most in a single CS chain, and the CS chain is a preferable structure that can induce monomerization and activation of PTP $\sigma$ (Figure 1). In contrast, with similar methods, we found that HS with one or more sulfate groups was sufficient to interact with PTP $\sigma$ (Sakamoto et al., 2019). These structures account for nearly half of the total HS in the injured CNS (Properzi et al., 2008). Again, the results imply that the HS chain is preferable for inducing multimerization and inactivation of the receptor. This idea was confirmed by a cell culture experiment and synthetic CS/HS oligosaccharides. Although how these two distinct GAG chains with different levels of sulfation interact with PTP $\sigma$ is still unclear, these data demonstrated that the frequency of the binding domain, defined by both the sulfation pattern and length in CS and HS, determined the action mode of each glycan on PTP $\sigma$.

To understand what a dystrophic endbulb is, and the consequence of PTP $\sigma$ activation by CS, we deeply observed adystrophic endbulb formed on a CS gradient in vitro by electron microscopy. We found that autophagosomes abnormally accumulated in the dystrophic endbulb. Immunostaining with LC3, a specific marker for autophagosome, supported the results. More importantly, autophagosomes also accumulated at the tips of the dissected corticospinal tract in a mouse model of SCI (Sakamoto et al., 2019).

Autophagy is an intracellular degradation system for organelles and cytoplasmic components (Mizushima and
Komatsu, 2011). Phagophore,a bowl-like membrane structure which is often formed at the contact site between endoplasmic reticulum and mitochondria,engulfs and encloses these components, producing autophagosome. The autophagosome then fuses with lysosome and is converted to autolysosome. As a consequence, components of autophagosome are delivered into lysosome and degraded by lysosomal enzymes. In axons, autophagy occurs preferentially at the axon tips and autophagosomes are retrogradely transported on microtubules to the cell body, fusing with lysosome during transport (Maday et al., 2012; Maday and Holzbaur, 2014). Because elongating axons have a high turnover of molecules and organelles including cytoskeleton, mitochondria, and membrane, the process seems to be essential to homeostasis for the elongation of axon tips. There are two mechanisms for the accumulation of autophagosomes (1) activation of autophagy and (2) disruption of autophagy flux, especially at the fusion step between autophagosomes and lysosomes. Immunocytochemical analysis using tandem-fluorescent LC3 (Kimura et al., 2007), which can distinguish between autophagosome and autolysosome, revealed that autophagy flux was severely disrupted in the dystrophic endbulb. Importantly, the knockdown of Syntaxin 17, Vamp 8, or Snap 29 by RNA interference, each of which is an essential soluble $N$-ethylmaleimide-sensitive factor attachment protein receptor (SNARE) for autophagosome-lysosome fusion (Itakura et al., 2012), successfully transformed healthy growth cones into dystrophic endbulb-like structures and significantly suppressed axonal elongation in cultured mouse dorsal root ganglion neuron. Consistent with this, treatments of the growth cones with chloroquine or bafilomycin Al, either of which inhibited the fusion between autophagosomes and lysosomes, gave similar results. Taken together, these results clearly demonstrated that disruption of autophagy flux was essential and sufficient for the formation of dystrophic endbulbs (Figure 1).

To link the PTP $\sigma$-autophagy axis, we focused on finding the specific substrate for PTP 6 that is involved at the fusion step between autophagosomes and lysosomes (Sakamoto et al., 2019). Cortactin is an actin-binding protein thatis required for the process (Lee et al., 2010; Hasegawa et al., 2016). It has several tyrosine phosphorylation sites, among which the tyrosine 421 and 466 phosphorylation sites are essential for its actin-stabilizing activity (Hasegawa et al., 2016). Some of these tyrosine-phosphorylated cortactins are specifically localized to the lysosome surface by the protein-lipid interaction (Hasegawa et al., 2016), where they provide stabilized actin fibers to lysosomes to fuse with autophagosomes. In the primary cultured dorsal root ganglion neurons on a CSPG gradient, reduced phosphorylation at tyrosine 421 of cortactin was observed in the dystrophic endbulb compared to healthy growth cones. Furthermore, silencing cortactin resulted in dystrophic endbulb formation, similar to the case with CS treatment. Thus, we conclude that CS binds to PTPo and the activated PTP $\sigma$ dephosphorylates cortactin. This results in the disruption of the completion of the autophagy flux, causing the transformation of the growth cone into a dormant dystrophic endbulb (Figure 1). 


\section{CONCLUDING REMARKS}

CS and HS, each with different backbones and sulfation patterns, can bind to РTP $\sigma$. Currently, it is well known that CS inhibits axonal regeneration while HS promotes it. In a recent study we prepared a library of HS octasaccharides and found that, through these octasaccharides together with CS octasaccharides, PTP $\sigma$ preferentially interacts with CS-E, a rare sulfation pattern in the natural CS chain, as well as with most HS oligomers bearing sulfate and sulfamate groups. Consequently, short and long stretches of natural CS and HS, respectively, bind to РТР $\sigma$. CS activates РТР $\sigma$, which dephosphorylates cortactin, a newly identified substrate for PTP $\sigma$, and disrupts autophagy flux at the autophagosomelysosome fusion step. The failure of autophagy flux causes an accumulation of autophagosomes at the growth cone and is sufficient to turn the growth cone into a dystrophic endbulb. As a result, we conclude that the sulfation patterns determine the length of the GAG segment that binds to PTP $\sigma$ and defines the fate of axonal regeneration through the PTP $\sigma$-cortactinautophagy axis. Our results shed light on the mechanisms by which GAGs function as ligands to cell surface receptors. In addition, the present findings provide a new therapeutic strategy, including glycomimetics. Indeed, we recently showed that enoxaparin, a heparin oligosaccharide medicine clinically used as an anticoagulant, promoted functional recovery in a

\section{REFERENCES}

Anderson, M. A., Burda, J. E., Ren, Y., Ao, Y., O’Shea, T. M., Kawaguchi, R., et al. (2016). Astrocyte scar formation aids central nervous system axon regeneration. Nature 532, 195-200. doi: 10.1038/nature17623

Aricescu, A. R., McKinnell, I. W., Halfter, W., and Stoker, A. W. (2002). Heparan sulfate proteoglycans are ligands for receptor protein tyrosine phosphatase sigma. Mol. Cell Biol. 22, 1881-1892. doi: 10.1128/mcb.22.6.1881-1892.2002

Asher, R. A., Morgenstern, D. A., and Fidler, P. S. (2000). Neurocan is upregulated in injured brain and in cytokine-treated astrocytes. J. Neurosci. 20, 2427-2438. doi: 10.1523/jneurosci.20-07-02427.2000

Benfey, M., and Aguayo, A. J. (1982). Extensive elongation of axons from rat brain into peripheral nerve grafts. Nature 296, 150-152. doi: 10.1038/296150a0

Bradbury, E. J., Moon, L. D., Popat, R. J., King, V. R., Bennett, G. S., Patel, P. N., et al. (2002). Chondroitinase ABC promotes functional recovery after spinal cord injury. Nature 416, 636-640. doi: 10.1038/416636a

Coles, C. H., Shen, Y., Tenney, A. P., Siebold, C., Sutton, G. C., Lu, W., et al. (2011). Proteoglycan-specific molecular switch for RPTP $\sigma$ clustering and neuronal extension. Science 332, 484-488. doi: 10.1126/science. 1200840

David, S., and Aguayo, A. J. (1981). Axonal elongation into peripheral nervous system "bridges" after central nervous system injury in adult rats. Science 214, 931-933. doi: 10.1126/science.6171034

Dickendesher, T. L., Baldwin, K. T., Mironova, Y. A., Koriyama, Y., Raiker, S. J., Askew, K. L., et al. (2012). NgR1 and NgR3 are receptors for chondroitin sulfate proteoglycans. Nat. Neurosci. 15, 703-712. doi: 10.1038/nn.3070

Fisher, D., Xing, B., Dill, J., Li, H., Hoang, H. H., Zhao, Z., et al. (2011). Leukocyte common antigen-related phosphatase is a functional receptor for chondroitin sulfate proteoglycan axon growth inhibitors. J. Neurosci. 31, 14051-14066. doi: 10.1523/JNEUROSCI.1737-11.2011

Frischknecht, R., Heine, M., Perrais, D., Seidenbecher, C. I., Choquet, D., and Gundelfinger, E. D. (2009). Brain extracellular matrix affects AMPA receptor lateral mobility and short-term synaptic plasticity. Nat. Neurosci. 12, 897-904. doi: $10.1038 / \mathrm{nn} .2338$ rat model of SCI, probably through the inactivation of PTP $\sigma$ (Ito et al., 2021).

We cannot exclude the possibility that other intracellular mediators and mechanisms are involved in the formation of dystrophic endbulbs and in the inhibition of axonal regeneration. For instance, we recently identified a lot of interactors, including cortactin, for PTP $\sigma$ by the proximity-dependent ligation assay (Gong et al., 2021). Further understanding toward dystrophic endbulbs is still needed to achieve complete regeneration of axons.

\section{AUTHOR CONTRIBUTIONS}

KS wrote the manuscript. KS, TO, and KK discussed the manuscript. All authors contributed to the article and approved the submitted version.

\section{FUNDING}

This work was supported by the Japan Society for the Promotion of Science (JSPS) KAKENHI Grant Nos. 19K07348 to KS and $19 \mathrm{H} 03415$ to KK, Grant from the Terumo Life Science Foundation to KS, and Grant from the Hori Science and Arts Foundation to KS.

Gogolla, N., Caroni, P., Lüthi, A., and Herry, C. (2009). Perineuronal nets protect fear memories from erasure. Science 325, 1258-1261. doi: 10.1126/science. 1174146

Gong, Y., Abudureyimu, S., Kadomatsu, K., and Sakamoto, K. (2021). Identification of PTPR $\sigma$-interacting proteins by proximity-labelling assay. J. Biochem. 169, 187-194. doi: 10.1093/jb/mvaa141

Hasegawa, J., Iwamoto, R., Otomo, T., Nezu, A., Hamasaki, M., and Yoshimori, T. (2016). Autophagosome-lysosome fusion in neurons requires INPP5E, a protein associated with Joubert syndrome. EMBO J. 35, 1853-1867. doi: 10. 15252/embj.201593148

Hughes, J. T. (1988). The Edwin Smith Surgical Papyrus: an analysis of the first case reports of spinal cord injuries. Paraplegia 26, 71-82. doi: 10.1038/sc.1988.15

Imagama, S., Sakamoto, K., Tauchi, R., Shinjo, R., Ohgomori, T., Ito, Z., et al. (2011). Keratan sulfate restricts neural plasticity after spinal cord injury. J. Neurosci. 31, 17091-17102. doi: 10.1523/jneurosci.5120-10.2011

Inatani, M., Irie, F., Plump, A. S., Tessier-Lavigne, M., and Yamaguchi, Y. (2003). Mammalian brain morphogenesis and midline axon guidance require heparan sulfate. Science 302, 1044-1046. doi: 10.1126/science.1090497

Itakura, E., Kishi-Itakura, C., and Mizushima, N. (2012). The hairpin-type tailanchored SNARE syntaxin 17 targets to autophagosomes for fusion with endosomes/lysosomes. Cell 151, 1256-1269. doi: 10.1016/j.cell.2012.11.001

Ito, S., Ozaki, T., Morozumi, M., Imagama, S., Kadomatsu, K., and Sakamoto, K. (2021). Enoxaparin promotes functional recovery after spinal cord injury by antagonizing PTPRo. Exp. Neurol. 340, 113679. doi: 10.1016/j.expneurol.2021. 113679

Ito, Z., Sakamoto, K., Imagama, S., Matsuyama, Y., Zhang, H., Hirano, K., et al. (2010). N-acetylglucosamine 6-O-sulfotransferase-1-deficient mice show better functional recovery after spinal cord injury. J. Neurosci. 30, 5937-5947. doi: 10.1523/JNEUROSCI.2570-09.2010

Johnson, K. G., Tenney, A. P., Ghose, A., Duckworth, A. M., Higashi, M. E., Parfitt, K., et al. (2006). The HSPGs Syndecan and Dallylike bind the receptor phosphatase LAR and exert distinct effects on synaptic development. Neuron 49, 517-531. doi: 10.1016/j.neuron.2006.01.026 
Kadomatsu, K., and Sakamoto, K. (2014). Sulfated glycans in network rewiring and plasticity after neuronal injuries. Neurosci. Res. 78, 50-54. doi: 10.1016/j.neures. 2013.10.005

Katagiri, Y., Morgan, A. A., Yu, P., Bangayan, N. J., Junka, R., and Geller, H. M. (2018). Identification of novel binding sites for heparin in receptor proteintyrosine phosphatase (RPTP $\sigma)$ : Implications for proteoglycan signaling. J. Biol. Chem. 293, 11639-11647. doi: 10.1074/jbc.RA118.003081

Kimura, S., Noda, T., and Yoshimori, T. (2007). Dissection of the autophagosome maturation process by a novel reporter protein, tandem fluorescent-tagged LC3. Autophagy 3, 452-460. doi: 10.4161/auto.4451

Lee, J. Y., Koga, H., Kawaguchi, Y., Tang, W., Wong, E., Gao, Y. S., et al. (2010). HDAC6 controls autophagosome maturation essential for ubiquitin-selective quality-control autophagy. EMBO J. 29, 969-980. doi: 10.1038/emboj.2009.405

Maday, S., and Holzbaur, E. L. (2014). Autophagosome biogenesis in primary neurons follows an ordered and spatially regulated pathway. Dev. Cell 30, 71-85. doi: 10.1016/j.devcel.2014.06.001

Maday, S., Wallace, K. E., and Holzbaur, E. L. (2012). Autophagosomes initiate distally and mature during transport toward the cell soma in primary neurons. J. Cell Biol. 196, 407-417. doi: 10.1083/jcb.201106120

Margolis, R. K., and Margolis, R. U. (1993). Nervous tissue proteoglycans. Experientia 49, 429-446. doi: 10.1007/bf01923587

Mikami, T., Yasunaga, D., and Kitagawa, H. (2009). Contactin-1 is a functional receptor for neuroregulatory chondroitin sulfate-E. J. Biol. Chem. 284, 44944499. doi: 10.1074/jbc.M809227200

Miyata, S., Komatsu, Y., Yoshimura, Y., Taya, C., and Kitagawa, H. (2012). Persistent cortical plasticity by upregulation of chondroitin 6-sulfation. Nat. Neurosci. 15, 414-422. doi: 10.1038/nn.3023

Mizushima, N., and Komatsu, M. (2011). Autophagy: renovation of cells and tissues. Cell 147, 728-741. doi: 10.1016/j.cell.2011.10.026

Moon, L. D., Asher, R. A., Rhodes, K. E., and Fawcett, J. W. (2001). Regeneration of CNS axons back to their target following treatment of adult rat brain with chondroitinase ABC. Nat. Neurosci. 4, 465-466. doi: 10. $1038 / 87415$

Pizzorusso, T., Medini, P., Berardi, N., Chierzi, S., Fawcett, J. W., and Maffei, L. (2002). Reactivation of ocular dominance plasticity in the adult visual cortex. Science 298, 1248-1251. doi: 10.1126/science.1072699

Pizzorusso, T., Medini, P., Landi, S., Baldini, S., Berardi, N., and Maffei, L. (2006). Structural and functional recovery from early monocular deprivation in adult rats. Proc. Natl. Acad. Sci. U S A 103, 8517-8522. doi: 10.1073/pnas.0602657103

Properzi, F., Carulli, D., Asher, R. A., Muir, E., Camargo, L. M., and van Kuppevelt, T. H. (2005). Chondroitin 6-sulphate synthesis is up-regulated in injured CNS, induced by injury-related cytokines and enhanced in axon-growth inhibitory glia. Eur. J. Neurosci. 21, 378-390. doi: 10.1111/j.1460-9568.2005.03876.x

Properzi, F., Lin, R., Kwok, J., Naidu, M., van Kuppevelt, T. H., and Ten Dam, G. B. (2008). Heparan sulphate proteoglycans in glia and in the normal and injured CNS: expression of sulphotransferases and changes in sulphation. Eur. J. Neurosci. 27, 593-604. doi: 10.1111/j.1460-9568.2008.06042.x

Rauch, U., Gao, P., Janetzko, A., Flaccus, A., Hilgenberg, L., Tekotte, H., et al. (1991). Isolation and characterization of developmentally regulated chondroitin sulfate and chondroitin/keratan sulfate proteoglycans of brain identified with monoclonal antibodies. J. Biol. Chem. 266, 14785-14801. doi: 10.1016/s00219258(18)98755-7

Rosenzweig, E. S., Salegio, E. A., Liang, J. J., Weber, J. L., Weinholtz, C. A., Brock, J. H., et al. (2019). Chondroitinase improves anatomical and functional outcomes after primate spinal cord injury. Nat. Neurosci. 22, 1269-1275. doi: 10.1038/s41593-019-0424-1
Ruschel, J., Hellal, F., Flynn, K. C., Dupraz, S., Elliott, D. A., Tedeschi, A., et al. (2015). Axonal regeneration. Systemic administration of epothilone B promotes axon regeneration after spinal cord injury. Science 348, 347-352. doi: 10.1126/ science.aaa2958

Sakamoto, K., and Kadomatsu, K. (2017). Mechanisms of axon regeneration: The significance of proteoglycans. Biochim. Biophys. Acta Gen. Subj. 1861, 2435-2441. doi: 10.1016/j.bbagen.2017.06.005

Sakamoto, K., Ozaki, T., Ko, Y. C., Tsai, C. F., Gong, Y., Morozumi, M., et al. (2019). Glycan sulfation patterns define autophagy flux at axon tip via PTPRsigmacortactin axis. Nat. Chem. Biol. 15, 699-709. doi: 10.1038/s41589-0190274-X

Shen, Y., Tenney, A. P., Busch, S. A., Horn, K. P., Cuascut, F. X., Liu, K., et al. (2009). PTPsigma is a receptor for chondroitin sulfate proteoglycan, an inhibitor of neural regeneration. Science 326, 592-596. doi: 10.1126/science. 1178310

Silver, J., and Miller, J. H. (2004). Regeneration beyond the glial scar. Nat. Rev. Neurosci. 5, 146-156. doi: 10.1038/nrn1326

Smith-Thomas, L. C., Fok-Seang, J., Stevens, J., Du, J. S., Muir, E., Faissner, A., et al. (1994). An inhibitor of neurite outgrowth produced by astrocytes. J. Cell Sci. 107(Pt 6), 1687-1695. doi: 10.1242/jcs.107.6.1687

Snow, D. M., Lemmon, V., Carrino, D. A., Caplan, A. I., and Silver, J. (1990). Sulfated proteoglycans in astroglial barriers inhibit neurite outgrowth in vitro. Exp. Neurol. 109, 111-130. doi: 10.1016/s0014-4886(05)80013-5

Snow, D. M., Watanabe, M., Letourneau, P. C., and Silver, J. (1991). A chondroitin sulfate proteoglycan may influence the direction of retinal ganglion cell outgrowth. Development 113, 1473-1485. doi: 10.1242/dev.113.4.1473

Tom, V. J., Steinmetz, M. P., Miller, J. H., Doller, C. M., and Silver, J. (2004). Studies on the development and behavior of the dystrophic growth cone, the hallmark of regeneration failure, in an in vitro model of the glial scar and after spinal cord injury. J. Neurosci. 24, 6531-6539. doi: 10.1523/JNEUROSCI.0994-04.2004

Tonks, N. K. (2006). Protein tyrosine phosphatases: from genes, to function, to disease. Nat. Rev. Mol. Cell Biol. 7, 833-846. doi: 10.1038/nrm2039

Tran, A. P., and Silver, J. (2021). Cathepsins in neuronal plasticity. Neural. Regen Res. 16, 26-35. doi: 10.4103/1673-5374.286948

Tran, A. P., Sundar, S., Yu, M., Lang, B. T., and Silver, J. (2018). Modulation of Receptor Protein Tyrosine Phosphatase Sigma Increases Chondroitin Sulfate Proteoglycan Degradation through Cathepsin B Secretion to Enhance Axon Outgrowth. J. Neurosci. 38, 5399-5414. doi: 10.1523/JNEUROSCI.3214-17. 2018

Wang, L., and Denburg, J. L. (1992). A role for proteoglycans in the guidance of a subset of pioneer axons in cultured embryos of the cockroach. Neuron 8 , 701-714. doi: 10.1016/0896-6273(92)90091-q

Warren, P. M., Steiger, S. C., Dick, T. E., MacFarlane, P. M., Alilain, W. J., and Silver, J. (2018). Rapid and robust restoration of breathing long after spinal cord injury. Nat. Commun. 9:4843. doi: 10.1038/s41467-018-06937-0

Conflict of Interest: The authors declare that the research was conducted in the absence of any commercial or financial relationships that could be construed as a potential conflict of interest.

Copyright (c) 2021 Sakamoto, Ozaki and Kadomatsu. This is an open-access article distributed under the terms of the Creative Commons Attribution License (CC BY). The use, distribution or reproduction in other forums is permitted, provided the original author(s) and the copyright owner(s) are credited and that the original publication in this journal is cited, in accordance with accepted academic practice. No use, distribution or reproduction is permitted which does not comply with these terms. 